\title{
Midventricular Ballooning Takotsubo Cardiomyopathy Complicated by Transient Complete Atrioventricular Block
}

\author{
Tomonori Sugiura ${ }^{1}$, Yasuaki Dohi ${ }^{1}$, Sumiyo Yamashita ${ }^{1}$, Toshihiko Goto ${ }^{1}$, Kenta Hachiya ${ }^{1}$, \\ Tomomitsu Tani ${ }^{1}$, Yoshimasa Murakami ${ }^{2}$ and Nobuyuki Ohte ${ }^{1}$
}

\begin{abstract}
A 63-year-old woman had mistakenly drunk detergent stored in a plastic bottle and was transported to our hospital via ambulance due to unconsciousness. In the emergency room, the monitoring electrocardiogram showed complete atrioventricular block and temporary pacing was thus started. Left ventriculography indicated midventricular Takotsubo cardiomyopathy, although coronary angiograms showed a normal appearance. The atrioventricular block was transient, and the reduced left ventricular wall motion gradually recovered. An electrophysiological study performed before discharge showed no abnormalities in the atrioventricular conduction system. In conclusion, we experienced a case of mid-ventricular Takotsubo cardiomyopathy complicated by transient complete atrioventricular block.
\end{abstract}

Key words: Takotsubo cardiomyopathy, midventricular ballooning, atrioventricular block, syncope

(Intern Med 52: 1919-1921, 2013)

(DOI: 10.2169/internalmedicine.52.0721)

\section{Introduction}

Takotsubo cardiomyopathy is characterized by apical ballooning with normal or increased contractility of the base of the left ventricle and is triggered by severe emotional stress (1-3). Although the physiological findings mimic acute myocardial infarction, the etiology of Takotsubo cardiomyopathy does not involve coronary artery disease. Importantly, the deterioration of the left ventricular function is reversible and normalizes within a few weeks or months. Although the underlying mechanism of Takotsubo cardiomyopathy is still not clearly understood, catecholamine surges in response to physiological or psychosomatic stress have been implicated in the onset of the disease (1-3). Recently, a few rare cases without typical apical ballooning have been reported $(4,5)$. We herein report a variant case of Takotsubo cardiomyopathy with midventricular ballooning complicated by complete atrioventricular (AV) block.

\section{Case Report}

A 63-year-old Japanese woman was transported to our hospital via ambulance due to loss of consciousness. She had mistakenly drunk detergent stored in a plastic bottle and became confused due to strong anxiety, followed by syncope and a gradual decline in her level of consciousness. She was unconscious on presentation to our emergency room, with a body temperature of $36.1{ }^{\circ} \mathrm{C}$, a blood pressure of $124 / 79$ $\mathrm{mmHg}$, a heart rate of $95 \mathrm{bpm}$ and a peripheral oxygen saturation of $98 \%$ under $2 \mathrm{~L} / \mathrm{min}$ of nasal oxygen. A plain chest X-ray showed an increased cardiothoracic ratio (58\%), and a 12-lead electrocardiogram demonstrated mild nonspecific changes in the ST segments and T waves (Fig. 1a). A venous blood gas analysis showed mixed acidosis with a high lactate level ( $\mathrm{pH}$ : 7.213, $\mathrm{PaCO}_{2}$ : $65.1 \mathrm{mmHg}, \mathrm{PaO}_{2}$ : $36.2 \mathrm{mmHg}, \mathrm{HCO}_{3}: 14 \mathrm{mM}$, lactate: $86.2 \mathrm{mg} / \mathrm{dL}$ ). Additional blood chemical analysis results were within the normal ranges. Brain magnetic resonance imaging with diffusion-weighted imaging was undertaken to exclude intracranial disease, which revealed no significant findings.

${ }^{1}$ Department of Cardio-Renal Medicine and Hypertension, Nagoya City University Graduate School of Medical Sciences, Japan and ${ }^{2}$ Devision of Cardiology, Nagoya City East Medical Center, Japan

Received for publication April 5, 2013; Accepted for publication April 22, 2013

Correspondence to Dr. Tomonori Sugiura, tomosugi@med.nagoya-cu.ac.jp 
a

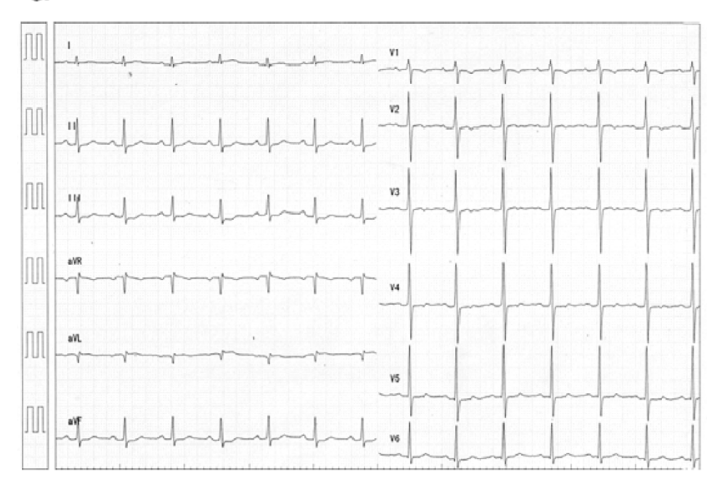

b

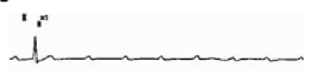

Figure 1. a: Twelve-lead electrocardiogram obtained on arrival. b: A strip of a monitor electrocardiogram recorded when the patient lost consciousness in the emergency room.

The patient had previously undergone surgery for congenital biliary dilatation due to pancreaticobiliary maljunction at 47 years of age. She had never smoked, did not report habitual alcohol drinking and had not been taking any medications.

During these examinations and laboratory investigations, the patient's consciousness level and command responsiveness gradually improved. However, she fell unconscious again in the emergency room, during which the monitoring electrocardiogram showed bradyarrythmia due to transient complete AV block (Fig. 1b). A subsequent echocardiogram demonstrated reduced left ventricular wall motion, despite an improvement of the complete AV block. A temporary pacing catheter was introduced via the internal jugular vein and placed in the right ventricle, followed by emergency cardiac catheterization. Although coronary angiograms indicated normal coronary arteries (Fig. 2a), the wall motion in the left ventricle was reminiscent of Takotsubo cardiomyopathy, i.e., the motion of the midventricular wall, but not the tip of the apex, was severely reduced (Fig. 2b). The intra-left ventricular end-diastolic pressure was $10 \mathrm{mmHg}$, and no systolic pressure gap was detected between the aorta and the left ventricle.

The patient was admitted to the intensive care unit, where her consciousness level gradually improved and she became alert without neurological deficits on the day following admission. Her blood gas analysis findings also returned to normal. Since the AV block was not recurrent, the temporary pacing catheter was removed on day 3 of intensive care admission. Her reduced left ventricular wall motion gradually recovered to within the normal limits after one week of admission. There were no laboratory data or image findings suggestive of pheochromocytoma or acute myocarditis, and electroencephalography showed no abnormal findings, such as those indicating epilepsy. Holter electrocardiogram monitoring and exercise stress testing demonstrated no ischemic changes or clinically significant arrhythmias, and the patient maintained normal durability for exercise.

On day 13, she underwent an electrophysiological study. The atrium-to-His bundle conduction time, His bundle-toventricular time and heart rate were $106 \mathrm{msec}, 50 \mathrm{msec}$ and 89 beats per minute, respectively. The response to burst pacing and single extra stimuli to the high right atrium with and without atropine sulfate $(0.5 \mathrm{mg} /$ body $)$ or disopyramide (47 mg/body) revealed normal cardiac conduction. Left ventriculography also showed a normal wall motion (Fig. 3). The patient was discharged on day 15 .

\section{Discussion}

Takotsubo cardiomyopahty often presents with abnormal findings on electrocardiography that mimic coronary artery disease and are occasionally complicated by various arrythmias $(2,6-8)$. Although the present patient did not exhibit typical ST-T changes or prolongation of the QT interval on the electrocardiograms, transient complete AV block was a complicating finding. Progressive impairment of AV conduction may have caused the decline in consciousness, syncope and peripheral circulatory failure. Indeed, syncope caused by complete AV block was observed in our emergency room. Several cases have been reported in which a permanent pacemaker was implanted due to bradyarrhythmia associated with Takotsubo cardiomyopathy that persisted even after improvements in left venticular wall motion $(7,8)$. However, the AV block observed in the present case was transient, and the electrophysiological study performed on day 13 showed normal findings, indicating that the AV block was functional and permanent pacemaker implantation was not indicated. The differences in the etiology of $\mathrm{AV}$ block between the present transient event and previously reported persistent cases remain unclear, although a concealed disorder in $\mathrm{AV}$ conduction present before the onset of Takotsubo cardiomyopathy may have contributed to the development of $\mathrm{AV}$ block in some cases (9). In contrast, an enhanced vagal nerve reflux relevant to extreme emotional stress could have induced the transient $\mathrm{AV}$ block observed in the present case (9). The burden of a vagal storm may have caused the functional AV block, which is compatible with the fact that the AV block was transient and no abnormalities were found on an electrophysiological study performed just before discharge.

Takotsubo cardiomyopathy often occurs in postmenopausal women with extreme catecholamine release induced by emotional stress $(2,3)$. Estrogen deficiency may cause increased sensitivity and responsiveness to catecholamine surges that then induce cardiac toxity and dysfunction independent of the anatomy of the coronary artery (3). In these respects, the present patient had a typical background for the onset of Takotsubo cardiomyopathy. Functional and/or metabolic brain disorders, such as epilepsy and electrolyte abnormalities, have also been reported to trigger Takotsubo cardiomyopathy $(10,11)$. However, none of these disorders were 
a

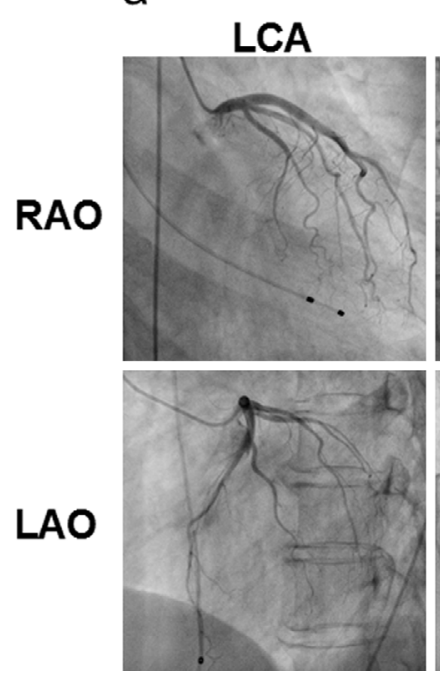

b

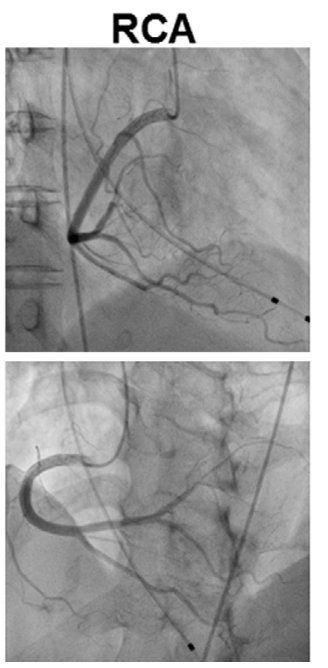

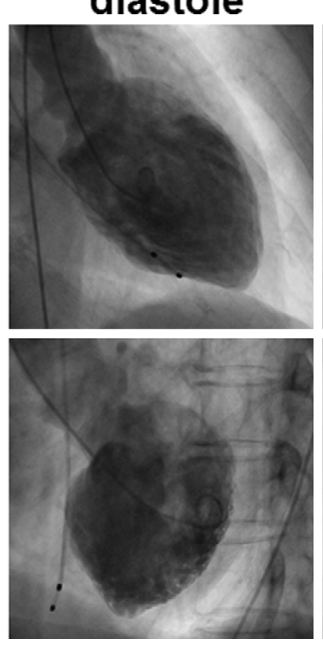

systole

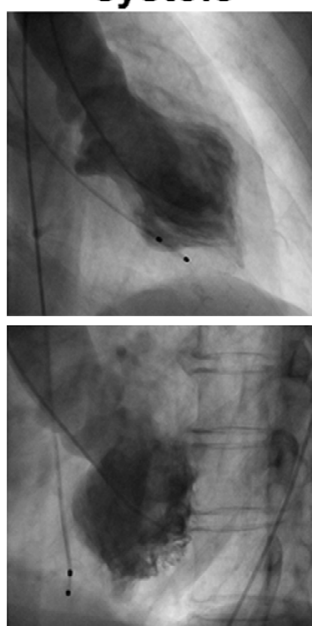

Figure 2. a: Coronary angiography performed in the acute phase. b: Left ventriculography performed in the acute phase. The left ventricular ejection fraction was $45 \%$ (the normal value in our hospital is over $55 \%$ ).

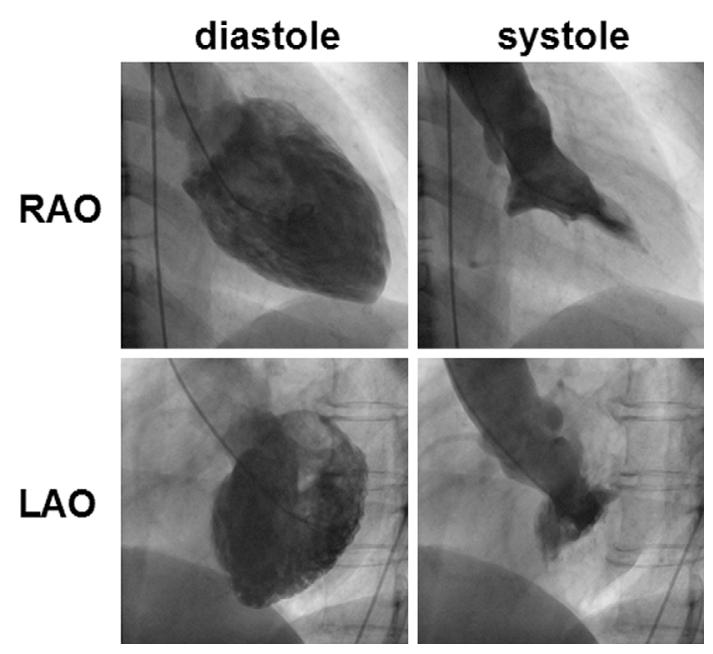

Figure 3. Left ventriculography performed before discharge. The left ventricular ejection fraction was $82 \%$ (the normal value in our hospital is over $55 \%$ ).

involved in this case based on the laboratory findings and results of brain magnetic resonance imaging and the electroencephalogram. Differences in the clinical characteristics or clinical course between patients with apical and midventricular ballooning Takotsubo cardiomyopathy have not yet been well described. However, Kurisu et al. reported that patients with midventricular ballooning tend to show limited changes on electrocardiography, consistent with the present findings in which typical ST-T segment changes were absent (12).

In conclusion, we encountered a rare case of midventricular Takotsubo cardiomyopathy complicated by syncope caused by a transient complete AV block in which the patient exhibited a gradual recovery without any associated se- quelae.

The authors state that they have no Conflict of Interest (COI).

\section{References}

1. Hurst RT 3rd, Prasad A, Askew JW, Sengupta PP, Tajik AJ. Takotsubo cardiomyopathy: a unique cardiomyopathy with variable ventricular morphology. JACC Cardiovasc Imaging 3: 641-649, 2010.

2. Kawai S, Kitabatake A, Tomoike H; Takotsubo Cardiomyopathy Group. Guidelines for diagnosis of takotsubo (ampulla) cardiomyopathy. Circ J 71: 990-992, 2007.

3. Balkin DM, Cohen LS. Takotsubo syndrome. Coron Artery Dis 22: 206-214, 2011.

4. Yasu T, Tone K, Kubo N, Saito M. Transient mid-ventricular ballooning cardiomyopathy: a new entity of Takotsubo cardiomyopathy. Int J Cardiol 110: 100-111, 2006.

5. Van de Walle SO, Gevaert SA, Gheeraert PJ, De Pauw M, Gillebert TC. Transient stress-induced cardiomyopathy with an "inverted takotsubo" contractile pattern. Mayo Clin Proc 81: 1499$1502,2006$.

6. Nef HM, Möllmann H, Sperzel J, et al. Temporary third-degree atrioventricular block in a case of apical ballooning syndrome. Int J Cardiol 113: e33-e35, 2006.

7. Inoue M, Kanaya H, Matsubara T, Uno Y, Yasuda T, Miwa K. Complete atrioventricular block associated with takotsubo cardiomyopathy. Circ J 73: 589-592, 2009.

8. Kodama S, Miyoshi K, Shiga Y, et al. Takotsubo cardiomyopathy complicated by high-grade atrioventricular block: A report of two cases. Exp Clin Cardiol 14: e35-e38, 2009.

9. Lee S, Wellens HJ, Josephson ME. Paroxysmal atrioventricular block. Heart Rhythm 6: 1229-1234, 2009.

10. Naganuma $M$, Isoda $K$, Ishizaki $M$, Ito $K$, Hirano $T$, Uchino $M$. Epilepsy and takotsubo cardiomyopathy: a case report. Intern Med 50: 2397-2399, 2011.

11. Kawano H, Matsumoto Y, Arakawa S, Hayano M, Fijisawa H. Takotsubo cardiomyopathy in a patient with severe hyponatremia associated with syndrome of inappropriate antidiuretic hormone. Intern Med 50: 727-732, 2011.

12. Kurisu S, Kato Y, Mitsuba N, et al. Comparison of electrocardiographic findings between the midventricular ballooning form and apical ballooning form of takotsubo cardiomyopathy. Clin Cardiol 34: 555-559, 2011.

(C) 2013 The Japanese Society of Internal Medicine http://www.naika.or.jp/imonline/index.html 